\title{
Half-linear eigenvalue problem: Limit behavior of the first eigenvalue for shrinking interval
}

\author{
Gabriella Bognár ${ }^{1}$ and Ondřej Došlýz $2^{*^{*}}$
}

\section{*Correspondence:}

dosly@math.muni.cz

${ }^{2}$ Department of Mathematics and

Statistics, Masaryk University,

Kotláŕská 2, Brno, 611 37, Czech

Republic

Full list of author information is

available at the end of the article

\begin{abstract}
We investigate the limit behavior of the first eigenvalue of the half-linear eigenvalue problem when the length of the interval tends to zero. We show that the important role is played by the limit behavior of ratios of primitive functions of coefficients in the investigated half-linear equation.

MSC: $34 C 10$
\end{abstract}

\section{Introduction}

We consider the eigenvalue problem associated with the half-linear second order differential equation

$$
-\left(r(t) \Phi\left(x^{\prime}\right)\right)^{\prime}+c(t) \Phi(x)=\lambda w(t) \Phi(x), \quad \Phi(x):=|x|^{p-2} x, \quad p>1,
$$

with $t \in(a, b),-\infty<a<b<\infty, r^{1-q}, c, w \in L^{1}(a, b), q=\frac{p}{p-1}$ being the conjugate exponent of $p$, and $r(t)>0, w(t)>0$. Equation (1) can be written as the first order system

$$
x^{\prime}=r^{1-q}(t) \Phi^{-1}(u), \quad u^{\prime}=(c(t)-\lambda w(t)) \Phi(x)
$$

$\Phi^{-1}(u)=|u|^{q-2} u$ being the inverse function of $\Phi$, and the integrability assumption on the functions $r^{1-q}, c, w$ implies the unique solvability of this system. The original paper of Elbert [1], where the existence and uniqueness results are proved via the half-linear version of the Prüfer transformation, deals with continuous functions in (2), but the idea of the proof applies without change to integrable coefficients when, as a solution $x, u$, absolutely continuous functions are considered (which satisfy (2) a.e. in $(a, b)$ ).

Along with (1), we consider the separated boundary conditions

$$
x(a) \cos _{p} \alpha-r^{q-1}(a) x^{\prime}(a) \sin _{p} \alpha=0, \quad x(b) \cos _{p} \beta-r^{q-1}(b) x^{\prime}(b) \sin _{p} \beta=0,
$$

where $\sin _{p}, \cos _{p}$ are the half-linear goniometric functions, which will be recalled in the next section. Motivated by the paper [2], where the linear Sturm-Liouville differential equation (which is the special case of (1)) is considered, we investigate the limit behavior (as $b \rightarrow a+$ ) of the first eigenvalue of (1), (3) in dependence on $\alpha, \beta$. We show that this 
limit behavior is, in a certain sense, the same as for an eigenvalue problem when boundary conditions (3) are associated with an equation with constant coefficients.

The investigation of half-linear eigenvalue problems is motivated, among others, by the fact that the partial differential equation with the $p$-Laplacian (which models, e.g., the flow of non-Newtonian fluids, while the linear case $p=2$ corresponds to the Newtonian fluid)

$$
-\Delta_{p} u+c(x) \Phi(u)=\lambda \Phi(u), \quad \Delta_{p}(u):=\operatorname{div}\left(\|u(x)\|^{p-2} \nabla u(x)\right), \quad x \in \mathbb{R}^{n},
$$

and the spherically symmetric potential $c$, can be reduced to an equation of the form (1). For this reason, motivated also by the linear case $p=2$, the problem of dependence of eigenvalues of (1), (3) on the functions $r, c, w$ and the boundary data $a, b, \alpha, \beta$ was a subject of the investigation in several recent papers. We refer to [3-6] and the references therein.

The paper is organized as follows. In the next section, we recall essentials of the qualitative theory of half-linear differential equations. Section 3 deals with the eigenvalue problem for an equation with constant coefficients. The main results of the paper, limit formulas for the first eigenvalue of (1), (3), are given in Section 4.

\section{Preliminaries}

First, we recall the concepts of half-linear goniometric functions. These functions, in the form presented here, appeared for the first time in [1]. In a modified form, they can also be found in other papers, e.g., in [7].

The half-linear sine function $\sin _{p}$ is defined as the solution of the differential equation

$$
\left(\Phi\left(x^{\prime}\right)\right)^{\prime}+(p-1) \Phi(x)=0
$$

given by the initial condition $x(0)=0, x^{\prime}(0)=1$. The function $\sin _{p}$ is $\pi_{p}$ anti-periodic (and hence $2 \pi_{p}$ periodic) with $\pi_{p}:=\frac{2 \pi}{p \sin (\pi / p)}$. The derivative $\left(\sin _{p} t\right)^{\prime}=: \cos _{p} t$ defines the halflinear cosine function. These functions satisfy the half-linear Pythagorean identity

$$
\left|\sin _{p} t\right|^{p}+\left|\cos _{p} t\right|^{p}=1
$$

The half-linear functions $\tan _{p}$ and $\cot _{p}$ are defined in a natural way as

$$
\tan _{p} t=\frac{\sin _{p} t}{\cos _{p} t}, \quad \cot _{p} t=\frac{\cos _{p} t}{\sin _{p} t}
$$

The inverse functions to these functions on $\left(-\pi_{p} / 2, \pi_{p} / 2\right)$ resp. $\left(0, \pi_{p}\right)$ are denoted by $\arctan _{p}$ and $\operatorname{arccot}_{p}$. By a direct computation, using (5) and the fact that (4) can be written as

$$
x^{\prime \prime}+\left|x^{\prime}\right|^{2-p} \Phi(x)=0,
$$

one can verify the formulas

$$
\left[\Phi\left(\cot _{p} t\right)\right]^{\prime}=-\frac{1}{\left|\sin _{p} t\right|^{p}}, \quad\left(\tan _{p} t\right)^{\prime}=\frac{1}{\left|\cos _{p} t\right|^{p}}, \quad\left(\arctan _{p} t\right)^{\prime}=\frac{1}{1+|t|^{p}} .
$$


The original proof of the unique solvability of (1) (and hence of (2), see [1]) is based on the half-linear version of the Prüfer transformation. Let $x$ be a nontrivial solution of (1), put

$$
x(t)=\rho(t) \sin _{p} \varphi(t), \quad r^{q-1}(t) x^{\prime}(t)=\rho(t) \cos _{p} \varphi(t) .
$$

Then the Prüfer angle $\varphi$ solves the first order differential equation

$$
\varphi^{\prime}=r^{1-q}(t)\left|\cos _{p} \varphi\right|^{p}+\frac{\lambda w(t)-c(t)}{p-1}\left|\sin _{p} \varphi\right|^{p} .
$$

The right-hand side of (7) is a Lipschitzian function with respect to $\varphi$, hence the standard existence, uniqueness, and continuous dependence on the initial data theory applies to this equation, and these results carry over via (6) to (2) and (1). Observe at this place that the right-hand side of (2) is not Lipschitzian, so this theory cannot be directly applied to (2).

The Prüfer transformation is closely associated with the Riccati-type differential equation

$$
v^{\prime}+\lambda w(t)-c(t)+(p-1) r^{1-q}(t)|v|^{q}=0,
$$

which is related to (1) by the Riccati substitution $v=r \Phi\left(x^{\prime} / x\right)$. The fact that we have in disposal a Riccati-type differential equation and the generalized Prüfer transformation implies that the linear oscillation theory extends almost verbatim to (1). In particular, similarly to the linear case, the eigenvalues of (1), (3) form an increasing sequence $\lambda_{n} \rightarrow \infty$, and the $n$th eigenfunction has exactly $n-1$ zeros in $(a, b)$, see [8] and also [9, Section 5.7]. For some recent references in this area, we refer to [3] and the references therein.

\section{Equation with constant coefficients}

In this section, as a motivation, we consider the equation

$$
-\left(\Phi\left(x^{\prime}\right)\right)^{\prime}=\lambda(p-1) \Phi(x)
$$

i.e., $r \equiv 1, c \equiv 0, w \equiv p-1$ in (1). The Riccati equation associated with (9) is

$$
v^{\prime}+(p-1)\left[\lambda+|v|^{q}\right]=0 .
$$

First, consider the case $\alpha=\beta \in\left(0, \pi_{p}\right)$ in (3). Then the first eigenfunction of (9), (3) corresponds to the solution of (10) satisfying

$$
v(a)=\Phi\left(\cot _{p} \alpha\right)=v(b) .
$$

To underline the dependence of eigenvalues of our eigenvalue problem on $b, \alpha, \beta$, we denote the first eigenvalue by $\lambda_{1}(b, \alpha, \beta)$. Also, when $\alpha=\beta$, we use the notation $\lambda_{1}(b, \alpha, \alpha)=$ : $\lambda_{1}(b, \alpha)$.

Obviously, the solution of (10) satisfying (11) is a constant solution when

$$
\lambda=\lambda_{1}(b, \alpha)=-\left|\Phi\left(\cot _{p} \alpha\right)\right|^{q}=-\left|\cot _{p} \alpha\right|^{p} .
$$


If $0<\alpha<\beta<\pi_{p}$, i.e., $v(a)=\Phi\left(\cot _{p} \alpha\right)>\Phi\left(\cot _{p} \beta\right)=v(b)$, we need $v$ to be decreasing. When the length of the interval $(a, b)$ tends to zero, $v^{\prime}(t)$ cannot be bounded from below in $(a, b)$, and hence

$$
\lambda_{1}(b, \alpha, \beta) \rightarrow \infty \quad \text { as } b \rightarrow a+.
$$

In the opposite case, when $0<\beta<\alpha<\pi_{p}$, i.e., $v(a)=\Phi\left(\cot _{p} \alpha\right)<\Phi\left(\cot _{p} \beta\right)=v(b)$, we need $v$ to be increasing, and using the same argument as before, we have $\lim _{b \rightarrow a+} \lambda_{1}(b, \alpha, \beta)=$ $-\infty$. Finally, if $\alpha=0$ and $\beta \in\left(0, \pi_{p}\right)$, we have $v(a+)=\infty$, and hence $\lim _{b \rightarrow a+} \lambda_{1}(b, 0, \beta)=\infty$, similarly, if $\beta=\pi_{p}$ and $\alpha \in\left(0, \pi_{p}\right)$, we have $\nu(b-)=-\infty$, and hence also $\lim _{b \rightarrow a+} \lambda \lambda_{1}(b, \alpha$, $\left.\pi_{p}\right)=\infty$.

The previous simple considerations are summarized in the next theorem.

Theorem 1 Let $\lambda_{1}(b, \alpha, \beta)$ denote the first eigenvalue of (9), (3) with $\alpha \in\left[0, \pi_{p}\right), \beta \in\left(0, \pi_{p}\right]$. Then

$$
\lim _{b \rightarrow a+} \lambda_{1}(b, \alpha, \beta)= \begin{cases}\infty & \text { if } \alpha<\beta, \\ -\left|\cot _{p} \alpha\right|^{p} & \text { if } \alpha=\beta, \\ -\infty & \text { if } \alpha>\beta\end{cases}
$$

Remark 1 In this section, for the sake of simplicity, we have considered a constant coefficients equation in the form (9), i.e., with $c=0, r=1$, and $w=p-1$. If the functions $r, c, w$ in (1) are constants equal to $r, c, w \in \mathbb{R}$, a slight modification of the previous considerations shows that for $\alpha=\beta$ we have

$$
\lambda_{1}(b, \alpha)=\frac{c}{w}-(p-1) \frac{r^{1-q}}{w}\left|\cot _{p} \alpha\right|^{p}
$$

(actually, this least eigenvalue does not depend on the endpoints $a, b$ ).

\section{Limit behavior of the first eigenvalue}

In this section, we consider general half-linear eigenvalue problem (1), (3). We denote

$$
R(t)=\int_{a}^{t} r^{1-q}(s) d s, \quad C(t)=\int_{a}^{t} c(s) d s, \quad W(t)=\int_{a}^{t} w(s) d s .
$$

In the next theorems, we discuss various asymptotic behavior of ratios of the functions $C$, $R, W$ for $t \rightarrow a+$, which implies various limit behavior of the first eigenvalue. The behavior of the higher eigenvalues is described at the end of this section.

In the proofs of the next theorems, given $\Lambda \in \mathbb{R}, \varphi(t)=\varphi(t, \Lambda)$, denotes the Prüfer angle of a solution $x$ of (1) with $\lambda=\Lambda$ satisfying (3) at $a$, i.e., $\varphi(a, \Lambda)=\alpha$.

We start with the most interesting case $\alpha=\beta$ in (3).

Theorem 2 Suppose that $\lim _{t \rightarrow a+} \frac{C(t)}{W(t)}=-\infty$. Then for any $\alpha \in\left(0, \pi_{p}\right)$, we have

$$
\lim _{b \rightarrow a+} \lambda_{1}(b, \alpha)=-\infty
$$


Proof We will show that $\lambda_{1}(b, \alpha)<\Lambda$ for any $\Lambda \in \mathbb{R}$ if $b>a$ is sufficiently close to $a$. Since the eigenfunction corresponding to the first eigenvalue $\lambda_{1}$ has no zero on $(a, b)$ (see, e.g., [10]), we can use the Riccati equation (8) for $v=\Phi\left(\cot _{p} \varphi\right)$ instead of equation (7) for $\varphi$. Using the mean-value theorem for Lebesgue integrals in computing the integral $\int_{a}^{b} r^{1-q}\left|\cot _{p} \varphi\right|^{p}$, integration of (8) gives

$$
\begin{aligned}
v(b)-v(a) & =\Phi\left(\cot _{p} \varphi(b)\right)-\Phi\left(\cot _{p} \alpha\right) \\
& =\left[\frac{C(b)}{W(b)}-\Lambda-(p-1) \frac{R(b)}{W(b)}\left|\cot _{p} \varphi\left(t_{b}\right)\right|^{p}\right] W(b),
\end{aligned}
$$

where $t_{b} \in(a, b)$. Hence, for $b$ sufficiently close to $a$,

$$
\Phi\left(\cot _{p} \varphi(b)\right)-\Phi\left(\cot _{p} \alpha\right) \leq\left(\frac{C(b)}{W(b)}-\Lambda\right) W(b)<0,
$$

i.e., $\varphi(b)>\alpha=\beta$. Thus, since $\varphi$ was the Prüfer angle corresponding to a solution of (1) with $\lambda=\Lambda$ satisfying (3) at $t=a$, we have $\lambda_{1}(b, \alpha)<\Lambda$ for the first eigenvalue when $b$ is in a sufficiently small right neighborhood of $a$ (since we need $\varphi(b)=\alpha$ for $\lambda=\lambda_{1}(b, \alpha)$ ). Therefore, (12) holds.

\section{Theorem 3 Suppose that}

$$
\limsup _{t \rightarrow a+} \frac{C(t)}{R(t)}<\infty, \quad \lim _{t \rightarrow a+} \frac{R(t)}{W(t)}=\infty,
$$

and let

$$
\alpha^{*}=\operatorname{arccot}_{p}\left(\max \left\{0, \frac{1}{p-1} \limsup _{t \rightarrow a+} \frac{C(t)}{R(t)}\right\}\right)^{1 / p} \in\left(0, \frac{\pi_{p}}{2}\right] .
$$

Then we have $\lambda_{1}(b, \alpha) \rightarrow-\infty$ as $b \rightarrow a+$ for any $\alpha \in\left(0, \pi_{p}\right) \backslash\left[\alpha^{*}, \pi_{p}-\alpha^{*}\right]$.

Proof Let $\alpha \in\left(0, \alpha^{*}\right) \cup\left(\pi_{p}-\alpha^{*}, \pi_{p}\right)$ be fixed, and take $\delta>0$ so small that

$$
(p-1)\left|\cot _{p} \alpha\right|^{p}>\limsup _{t \rightarrow a+} \frac{C(t)}{R(t)}+2 \delta .
$$

Such a positive $\delta$ exists according to the definition of the number $\alpha^{*}$. Formula (13) implies that for $\tau$ sufficiently close to $\alpha$, we have the inequality

$$
(p-1)\left|\cot _{p} \tau\right|^{p}>\frac{C(t)}{R(t)}+\delta
$$

when $t$ is sufficiently close to $a$. Again, integration of (8), together with the mean value theorem applied to the last integral on the right-hand side of (8), gives

$$
\begin{aligned}
& \Phi\left(\cot _{p} \varphi(b)\right)-\Phi\left(\cot _{p} \alpha\right) \\
& \quad=\left[\frac{R(b)}{W(b)}\left(\frac{C(b)}{R(b)}-(p-1)\left|\cot _{p} \varphi\left(t_{b}\right)\right|^{p}\right)-\Lambda\right] W(b)<0 .
\end{aligned}
$$


Hence $\varphi(b)>\alpha$. This implies that $\lambda_{1}(b, \alpha)<\Lambda$ for every $\Lambda \in \mathbb{R}$, and thus, $\lambda_{1}(b, \alpha) \rightarrow-\infty$ as $b \rightarrow a+$.

Theorem 4 Assume that

$$
\liminf _{t \rightarrow a+} \frac{C(t)}{R(t)}>0 \text { and } \lim _{t \rightarrow a+} \frac{C(t)}{W(t)}=\infty .
$$

Let

$$
\alpha^{*}=\operatorname{arccot}_{p}\left(\frac{1}{p-1} \liminf _{t \rightarrow a+} \frac{C(t)}{R(t)}\right)^{1 / p} \in\left[0, \frac{\pi_{p}}{2}\right) .
$$

Then we have $\lambda_{1}(b, \alpha) \rightarrow \infty$ as $b \rightarrow a+$ for any $\alpha \in\left(\alpha^{*}, \pi_{p}-\alpha^{*}\right)$.

Proof The first formula in (14) implies that $C(t)>0$ for $t$ in a right neighborhood of $a$. Take $\alpha \in\left(\alpha^{*}, \pi_{p}-\alpha^{*}\right)$, and let $\delta>0$ be so small that

$$
(1-2 \delta) \liminf _{t \rightarrow a+} \frac{C(t)}{R(t)}>(p-1)\left|\cot _{p} \alpha\right|^{p} .
$$

Again, such $\delta>0$ exists according to the definition of $\alpha^{*}$. Hence, for $\tau$ sufficiently close to $\alpha$, from (16), we have for $t$ close to $a$ that

$$
(1-\delta) \frac{C(t)}{R(t)}>(p-1)\left|\cot _{p} \tau\right|^{p}
$$

Let $\Lambda \in \mathbb{R}$ be arbitrary. Similarly as in the proof of the previous theorem,

$$
\begin{aligned}
& \Phi\left(\cot _{p} \varphi(b)\right)-\Phi\left(\cot _{p} \alpha\right) \\
& \quad=\left[\frac{C(b)}{W(b)}\left(1-(p-1) \frac{R(b)}{C(b)}\left|\cot _{p} \varphi\left(t_{b}\right)\right|^{p}\right)-\Lambda\right] W(b)>0
\end{aligned}
$$

if $b$ is sufficiently close to $a$, i.e., $\varphi(b)<\alpha$, and hence $\lambda_{1}(b, \alpha)>\Lambda$. Therefore, $\lim _{b \rightarrow a+} \lambda_{1}(b$, $\alpha)=\infty$.

\section{Theorem 5 Assume that}

$$
\limsup _{t \rightarrow a+} \frac{R(t)}{W(t)}<\infty
$$

If

$$
\lim _{t \rightarrow a+}\left(\frac{C(t)}{W(t)}-(p-1) \frac{R(t)}{W(t)}\left|\cot _{p} \varphi\right|^{p}\right)=L \in \mathbb{R} \cup\{ \pm \infty\},
$$

then

$$
\lim _{b \rightarrow a+} \lambda_{1}(b, \alpha)=L .
$$


Proof Let $\Lambda \in \mathbb{R}$ be arbitrary. Similarly as in the previous theorems,

$$
\begin{aligned}
\Phi\left(\cot _{p} \varphi(b)\right)-\Phi\left(\cot _{p} \alpha\right) \\
=\left[\frac{C(b)}{W(b)}-(p-1) \frac{R(b)}{W(b)}\left|\cot _{p} \alpha\right|^{p}\right. \\
\left.\quad-\Lambda+(p-1) \frac{R(b)}{W(b)}\left(\left|\cot _{p} \alpha\right|^{p}-\left|\cot _{p} \varphi\left(t_{b}\right)\right|^{p}\right)\right] W(b) .
\end{aligned}
$$

If $L=-\infty$, the expression in line (17) tends to $-\infty$ as $b \rightarrow a+$ while remaining terms on the right-hand side of the previous formula are bounded. Hence the expression on the righthand side is negative for $b$ close to $a$, which means that $\varphi(b)>\alpha$ for these $b$. However, this implies that $\lambda_{1}(b, \alpha)<\Lambda$ in a right neighborhood of $a$, and since $\Lambda$ was arbitrary, we have $\lim _{b \rightarrow a} \lambda_{1}(b, \alpha)=-\infty$. The same arguments imply that $\lim _{b \rightarrow a+} \lambda_{1}(b, \alpha)=\infty$ if $L=+\infty$.

Finally, if $L \in \mathbb{R}$, take first $\Lambda=L+\varepsilon$. Since the last term in (18) tends to zero as $b \rightarrow a+$, we obtain using the same argument as in the previous part of the proof that $\lambda_{1}(b, \alpha)<L+\varepsilon$ for $b$ sufficiently close to $a$. Taking $\Lambda=L-\varepsilon$, we obtain $\lambda_{1}(b, \alpha)>L-\varepsilon$ for $b$ in a right neighborhood of $a$, and this completes the proof.

Theorem 6 Suppose that

$$
\lim _{t \rightarrow a+} \frac{R(t)}{W(t)}<\infty
$$

and $\alpha \in\left(0, \pi_{p}\right)$. If

$$
\lim _{b \rightarrow a^{+}} \lambda_{1}(b, \alpha)=L \quad \text { exists finite, }
$$

then

$$
\lim _{t \rightarrow a+}\left(\frac{C(t)}{W(t)}-(p-1) \frac{R(t)}{W(t)}\left|\cot _{p} \alpha\right|^{p}\right)=L .
$$

Proof Denote $\lambda(b):=\lambda_{1}(b, \alpha)$, and let $\varphi(t, \lambda(b))$ be the Prüfer angle of the solution $x$ of (1) with $\lambda=\lambda(b)=\lambda_{1}(b, \alpha)$ satisfying (3) with $\alpha=\beta$. Then

$$
\begin{aligned}
0= & \Phi\left(\cot _{p} \beta\right)-\Phi\left(\cot _{p} \alpha\right) \\
= & \Phi\left(\cot _{p} \varphi(b, \lambda(b))\right)-\Phi\left(\cot _{p} \varphi(a, \lambda(b))\right) \\
= & C(b)-\lambda(b) W(b)-(p-1) R(b)\left|\cot _{p} \alpha\right|^{p} \\
& +(p-1) R(b)\left(\left|\cot _{p} \alpha\right|^{p}-\left|\cot _{p} \varphi\left(t_{b}, \lambda(b)\right)\right|^{p}\right) \\
= & {\left[\frac{C(b)}{W(b)}-(p-1) \frac{R(b)}{W(b)}\left|\cot _{p} \alpha\right|^{p}\right.} \\
& \left.-\lambda(b)+(p-1) \frac{R(b)}{W(b)}\left(\left|\cot _{p} \alpha\right|^{p}-\left|\cot _{p} \varphi\left(t_{b}, \lambda(b)\right)\right|^{p}\right)\right] W(b)=0
\end{aligned}
$$

for some $t_{b} \in(a, b)$. Therefore, from (21), (22), we obtain

$$
\frac{C(b)}{W(b)}-(p-1) \frac{R(b)}{W(b)}\left|\cot _{p} \alpha\right|^{p}=\lambda(b)-(p-1) \frac{R(b)}{W(b)}\left(\left|\cot _{p} \alpha\right|^{p}-\left|\cot _{p} \varphi\left(t_{b}, \lambda(b)\right)\right|^{p}\right) .
$$


Letting $b \rightarrow a+$ and using that

$$
\lim _{t \rightarrow a+} \lambda(b)=\lim _{b \rightarrow a+} \lambda_{1}(b, \alpha)=L \in \mathbb{R}
$$

(which means that $\lambda(b)$ is bounded in a neighborhood of $a$ ), we see that

$$
\lim _{b \rightarrow a+} \varphi\left(t_{b}, \lambda(b)\right)=\alpha,
$$

and (20) is proved.

Theorem 7 If $\lim _{b \rightarrow a+} \lambda_{1}(b, \alpha)$ has a finite value for two different values of $\alpha_{1}$ and $\alpha_{2}$ with $\alpha_{1}, \alpha_{2} \in\left(0, \pi_{p}\right)$ and $\alpha_{1} \neq \pi_{p}-\alpha_{2}$, then

$$
\lim _{t \rightarrow a+} \frac{R(t)}{W(t)} \quad \text { and } \quad \lim _{t \rightarrow a+} \frac{C(t)}{W(t)}
$$

exist finite, and for each $\alpha \in\left(0, \pi_{p}\right)$, we have

$$
\lim _{b \rightarrow a+} \lambda_{1}(b, \alpha)=\lim _{t \rightarrow a+} \frac{C(t)}{W(t)}-(p-1)\left|\cot _{p} \alpha\right|^{p} \lim _{t \rightarrow a+} \frac{R(t)}{W(t)} .
$$

Proof First of all, we have

$$
\left|\cot _{p} \alpha_{1}\right|^{p} \neq\left|\cot _{p} \alpha_{2}\right|^{p}
$$

Denote $\lambda_{1}\left(b, \alpha_{i}\right)=: \mu_{i}(b)$ for $i=1,2$, and let $\varphi\left(t, \mu_{i}(b)\right)$ be the Prüfer angle of the solution of (1), (3) with $\lambda=\mu_{i}(b)$ and $\alpha=\beta$. Since $\varphi\left(b, \mu_{i}(b)\right)=\varphi\left(a, \mu_{i}(b)\right)$, we have from (8)

$$
C(b)-\mu_{i}(b) W(b)-(p-1) R(b)\left|\cot _{p} \varphi\left(t_{i, b}, \mu_{i}(b)\right)\right|^{p}=0, \quad i=1,2,
$$

where $t_{i, b} \in(a, b)$. Hence, one gets

$$
\frac{C(b)}{W(b)}-(p-1) \frac{R(b)}{W(b)}\left|\cot _{p} \varphi\left(t_{i, b}, \mu_{i}(b)\right)\right|^{p}=\mu_{i}(b), \quad i=1,2
$$

Subtracting these two equations and using the fact that

$$
\left|\cot _{p} \varphi\left(t_{1, b}, \mu_{1}(b)\right)\right| \neq\left|\cot _{p} \varphi\left(t_{2, b}, \mu_{2}(b)\right)\right|
$$

for $b$ sufficiently close to $a$ (this follows from (24)), we have

$$
\frac{R(b)}{W(b)}=\frac{\mu_{1}(b)-\mu_{2}(b)}{\left|\cot _{p} \varphi\left(t_{1, b}, \mu_{1}(b)\right)\right|^{p}-\left|\cot _{p} \varphi\left(t_{2, b}, \mu_{2}(b)\right)\right|^{p}} \rightarrow \frac{\lambda_{1}\left(a+, \alpha_{1}\right)-\lambda_{1}\left(a+, \alpha_{2}\right)}{\left|\cot _{p} \alpha_{1}\right|^{p}-\left|\cot _{p} \alpha_{2}\right|^{p}}
$$

as $b \rightarrow a+$, so $\lim _{b \rightarrow a+} \frac{R(b)}{W(b)}$ exists finite, and hence from (25), the same holds for $\lim _{b \rightarrow a+} \frac{C(b)}{W(b)}$, and the conclusion follows from Theorem 5 .

We finish the paper with a brief treatment of the case $\alpha \neq \beta$ in (3). We show that the situation is similar as in the case of a constant coefficients equation treated in Section 3. 
Theorem 8 Let $\alpha \in\left[0, \pi_{p}\right), \beta \in\left(0, \pi_{p}\right]$.

(i) If $\alpha<\beta$, then

$$
\lim _{b \rightarrow a+} \lambda_{1}(b, \alpha, \beta)=\infty
$$

(ii) If $\alpha>\beta$, then

$$
\lim _{b \rightarrow a+} \lambda_{1}(b, \alpha, \beta)=-\infty
$$

Proof We will prove the part (i) only, the proof of (ii) is analogical. Let $\Lambda \in \mathbb{R}$ be arbitrary, and let $\varphi(t, \Lambda)$ be the Prüfer angle of the solution $x$ of (1) with $\lambda=\Lambda$ satisfying (3) at $t=a$. Since $\varphi$ is a continuous function of $t$, and $\varphi(a, \Lambda)=\alpha<\beta$, we have $\varphi(b, \Lambda)<\beta$ if $b$ is sufficiently close to $a$. Hence, using the same argument as before, we have $\lambda_{1}(b, \alpha, \beta)>\Lambda$, which implies (26).

Remark 2 Until now, we have considered the first eigenvalue $\lambda_{1}(b, \alpha, \beta)$ only. Concerning the asymptotic behavior of higher eigenvalues $\lambda_{n}(b, \alpha, \beta)$, we have

$$
\lim _{b \rightarrow a+} \lambda_{n}(b, \alpha, \beta)=\infty
$$

for any $\alpha \in\left[0, \pi_{p}\right), \beta \in\left(0, \pi_{p}\right]$. This formula follows in the case $\alpha<\beta$ from the general theory of half-linear eigenvalues problem (see $[8,10])$, which says that the eigenvalues form an increasing sequence tending to $\infty$, and from the fact that $\lim _{b \rightarrow a+} \lambda_{1}(b, \alpha, \beta)=\infty$. If $\alpha>\beta$, then $\cot _{p} \alpha<\cot _{p} \beta$, but for $n \in \mathbb{N}$, we have $\alpha<\beta+n \pi_{p}$ and higher eigenvalues correspond to the situation when the Prüfer angle of a solution of (1) satisfying the first condition in (3) satisfies $\varphi(b)=\beta+n \pi_{p}$. Hence, the growth of $\varphi$ must be unbounded when $b \rightarrow a+$, and hence (27) holds also for $\alpha>\beta$.

\footnotetext{
Competing interests

The authors declare that they have no competing interests.
}

\section{Authors' contributions}

The work presented here was carried out in collaboration between the authors. The authors contributed to every part of this study equally and read and approved the final version of the manuscript.

\section{Author details}

${ }^{1}$ University of Miskolc, Egyetemváros, Miskolc, 3515, Hungary. ${ }^{2}$ Department of Mathematics and Statistics, Masaryk University, Kotlářská 2, Brno, 611 37, Czech Republic.

\section{Acknowledgements}

The research of the first author was carried out as a part of the TAMOP-4.2.2/B-10/1-2010-0008 project in the framework of the New Hungarian Development Plan. The realization of this project is supported by the European Union and co-financed by the European Social Fund. The second author was supported by the Grant GAP 201/11/0768 of the Czech Grant Agency.

Received: 27 June 2013 Accepted: 4 September 2013 Published: 07 Nov 2013

\section{References}

1. Elbert, Á: A half-linear second order differential equation. In: Qualitative Theory of Differential Equations, Vol. I, II (Szeged, 1979). Colloq. Math. Soc. János Bolyai, vol. 30, pp. 153-180. North-Holland, Amsterdam (1981)

2. Kong, Q, Wu, H, Zettl, A: Limit of Sturm-Liouville eigenvalues when the interval shrinks to an end point. Proc. R. Soc. Edinb., Sect. A 138, 323-338 (2008)

3. Benedikt, J, Drábek, P: Estimates of the principal eigenvalue of the $p$-Laplacian. J. Math. Anal. Appl. 393, 311-315 (2012) 
4. Binding, PA, Drábek, P: Sturm-Liouville theory for the p-Laplacian. Studia Sci. Math. Hung. 40, 375-396 (2003)

5. Kong, L, Kong, Q: Right-definite half-linear Sturm-Liouville problems. Proc. R. Soc. Edinb. A 137, 77-92 (2007)

6. Kusano, T, Naito, M, Tanigawa, T: Second-order half-linear eigenvalue problems. Fukuoka Univ. Sci. Rep. 27, 1-7 (1997)

7. Lindqvist, P: Some remarkable sine and cosine functions. Ric. Mat. 44, 260-290 (1995)

8. Kusano, T, Naito, M: Sturm-Liouville eigenvalue problems for half-linear ordinary differential equations. Rocky Mt. J. Math. 31, 1039-1054 (2001)

9. Došlý, O, Řehák, P: Half-Linear Differential Equations. North-Holland Mathematics Studies, vol. 202. Elsevier, Amsterdam (2005)

10. Eberhard, W, Elbert, Á: On the eigenvalues of half-linear boundary value problems. Math. Nachr. 183, 55-72 (1997)

10.1186/1687-2770-2013-221

Cite this article as: Bognár and Došlý: Half-linear eigenvalue problem: Limit behavior of the first eigenvalue for shrinking interval. Boundary Value Problems 2013, 2013:221

\section{Submit your manuscript to a SpringerOpen ${ }^{\circ}$ journal and benefit from:}

- Convenient online submission

- Rigorous peer review

- Immediate publication on acceptance

- Open access: articles freely available online

- High visibility within the field

- Retaining the copyright to your article 